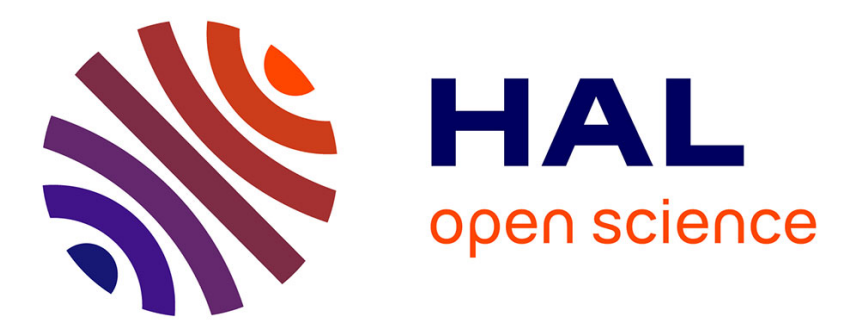

\title{
LOCAL EXISTENCE AND GLOBAL NONEXISTENCE OF SOLUTION FOR LOVE-EQUATION WITH INFINITE MEMORY
}

Khaled Zennir

\section{- To cite this version:}

Khaled Zennir. LOCAL EXISTENCE AND GLOBAL NONEXISTENCE OF SOLUTION FOR LOVE-EQUATION WITH INFINITE MEMORY. 2018. hal-01910485

\section{HAL Id: hal-01910485 \\ https://hal.science/hal-01910485}

Preprint submitted on 1 Nov 2018

HAL is a multi-disciplinary open access archive for the deposit and dissemination of scientific research documents, whether they are published or not. The documents may come from teaching and research institutions in France or abroad, or from public or private research centers.
L'archive ouverte pluridisciplinaire HAL, est destinée au dépôt et à la diffusion de documents scientifiques de niveau recherche, publiés ou non, émanant des établissements d'enseignement et de recherche français ou étrangers, des laboratoires publics ou privés. 


\title{
LOCAL EXISTENCE AND GLOBAL NONEXISTENCE OF SOLUTION FOR LOVE-EQUATIONS WITH INFINITE MEMORY
}

\author{
KHALED ZENNIR
}

\begin{abstract}
In this paper, we consider an IBVP for a nonlinear Love equation with infinite memory. By combining the linearization method, the FaedoGalerkin method and the weak compactness method, we prove the local existence and uniqueness of weak solution. The finite time blow up of weak solution is considered.
\end{abstract}

\section{INTRODUCTION}

Many interesting physical phenomena in which delay effects occur (e.g., population dynamics) can be modeled by partial differential equations with finite or infinite visco-elastic memory which provides a typical damping mechanism in nature. The well-posedness and stability for elasticity and visco-elasticity systems attracted lots of interests in recent years, where different types of dissipative mechanisms have been introduced to obtain diverse results. In general, the stability properties of visco-elastic system are in dependence on the form of the convolution kernel (see in this direction results in [2], 3], [18, [19], 20], 21], 27], 33], [34, [35, ...). The blow up is an essential and very important phenomena to be study in the evolution PDEs, there is a different between global nonexistence which means that the local solution can't be continue to exist in time i.e. there exists a finite time blow up which is our case in this paper and the blowing up $\forall t>0$, that is the solution goes to infinity for all $t>0$. Blow up an effect occurs, for example, when a sea wave tumbles to the shore, when a computer breaks down as a result of electrical breakdown, when a nuclear bomb explodes and in a number of other interesting physical phenomena. (see [1, [4, [12], 18], [19], 24], 25], [26], 32], 33], .. )

1.1. Formulation of problem. Denote $u=u(x, t), u^{\prime}=u_{t}=\frac{\partial u}{\partial t}(x, t), u^{\prime \prime}=$ $u_{t t}=\frac{\partial^{2} u}{\partial t^{2}}(x, t), u_{x}=\frac{\partial u}{\partial x}(x, t), u_{x x}=\frac{\partial^{2} u}{\partial x^{2}}(x, t)$. In this article, we consider the Love-equation in the form

$$
\begin{aligned}
& u^{\prime \prime}-\left(\lambda_{0} u_{x}+\lambda_{1} u_{x}^{\prime}+u_{x}^{\prime \prime}\right)_{x}+\lambda \int_{-\infty}^{t} g(t-s) u_{x x}(s) d s \\
& =F[u]-(F[u])_{x}+f(x, t), \quad x \in \Omega=(0,1), 0<t<T,
\end{aligned}
$$

where

$$
F[u]=F\left(x, t, u, u_{x}, u^{\prime}, u_{x}^{\prime}\right) \in C^{1}\left([0,1] \times \mathbb{R}^{+} \times \mathbb{R}^{4}\right),
$$

and $\lambda, \lambda_{0}, \lambda_{1}>0$, are constants. The given functions $g, f$ are specified later. With $F=F\left(x, t, y_{1}, \ldots, y_{4}\right)$, we put $D_{1} F=\frac{\partial F}{\partial x}, D_{2} F=\frac{\partial F}{\partial t}, D_{i+2} F=\frac{\partial F}{\partial y_{i}}$, with $i=$

2010 Mathematics Subject Classification. 35L20, 35L70, 37B25, 93D15.

Key words and phrases. Nonlinear Love-equation; Local Existence; Blow-up; Infinite Memory. 
$1, \ldots, 4$.

Equation (1.1) satisfies the homogeneous Dirichlet boundary conditions:

$$
u(0, t)=u(1, t)=0, \quad t>0,
$$

and the following initial conditions

$$
u(x,-t)=u_{0}(x, t), \quad u^{\prime}(x, 0)=u_{1}(x) .
$$

To deal with a wave equation with infinite history, we assume that the kernel function $g$ satisfies the following hypothesis:

(Hyp1:) $g: \mathbb{R}^{+} \rightarrow \mathbb{R}^{+}$is a non-increasing $C^{1}$ function such that

$$
\lambda_{0}-\lambda \int_{0}^{\infty} g(s) d s=l>0, \quad g(0)>0 .
$$

We need the following assumptions on source forces:

(Hyp2:) $u_{0}(0), u_{1} \in H_{0}^{1} \cap H^{2}$;

(Hyp3:) $f \in H^{1}((0,1) \times(0, T))$;

(Hyp4:) $F \in C^{1}\left([0,1] \times[0, T] \times \mathbb{R}^{4}\right)$, such that $F\left(0, t, 0, y_{2}, 0, y_{4}\right)=F\left(1, t, 0, y_{2}, 0, y_{4}\right)=$ 0 for all $t \in[0, T], \quad y_{2}, y_{4} \in \mathbb{R}$.

1.2. Bibliographical notes. We start our literature review concerning visco-elastic problems with the pioneer work of Dafermos [10], where the author considered a one-dimensional visco-elastic problem

$$
\rho u^{\prime \prime}=c u_{x x}-\int_{-\infty}^{t} g(t-s) u_{s s} d s,
$$

and established various existence results and then proved, for smooth monotone decreasing relaxation functions, that the solutions go to zero as t goes to infinity. In [13, Hrussa considered a one-dimensional nonlinear visco-elastic equation

$$
u^{\prime \prime}=c u_{x x}-\int_{0}^{t} m(t-s)\left(\phi\left(u_{x}\right)\right)_{x} s d s=f,
$$

and proved several global existence results for large data. Here, the author also obtained a decay rate of solution. Messaoudi in [18] investigated the $\mathrm{u}=$ interaction between the nonlinear damping and nonlinear source in the equation

$$
u^{\prime \prime}=\Delta u+\int_{0}^{t} g(t-s) \Delta u d s+a u^{\prime}\left|u^{\prime}\right|^{m}=b u|u|^{\gamma},
$$

and showed, under suitable conditions on $g$, that solutions with negative energy blow up in finite time if $\gamma>m$, and continue to exist if $m>\gamma$.

Concerning problems with infinite history, we mention the work 11 in which considered the following semi-linear hyperbolic equation, in a bounded domain of $\mathbb{R}^{3}$,

$$
u^{\prime \prime}-K(0) \Delta u-\int_{0}^{\infty} K^{\prime}(s) \Delta u(t-s) d s+g(u)=f,
$$

with $K(0), K(\infty)>0, K^{\prime} \leq 0$ and gave the existence of global attractors for the problem. Next in 33, proved that the solutions of a system of wave equations with visco-elastic term, degenerate damping, and strong nonlinear sources acting in both equations at the same time are globally non-existing provided that the initial data are sufficiently large in a bounded domain; the initial energy is positive, and the 
strongly nonlinear functions $f_{1}$ and $f_{2}$ located in the sources satisfy an appropriate conditions. The author concentrate his studies on the role of the nonlinearities of sources. After that, in [31, the authors considered a fourth-order suspension bridge equation with nonlinear damping term and source term. The authors gave necessary and sufficient condition for global existence and energy decay results without considering the relation between $m$ and $p$. Moreover, when $p>m$, they gave sufficient condition for finite time blow-up of solutions. The lower bound of the blow-up time is also established.

Recently, in [26], the authors studied a three-dimensional (3D) visco-elastic wave equation with nonlinear weak damping, supercritical sources and prescribed past history, $t \leq 0$ in

$$
u^{\prime \prime}-k(0) \Delta u-\int_{0}^{\infty} k^{\prime}(s) \Delta u(t-s) d s+\left|u^{\prime}\right|^{m-1} u^{\prime}=|u|^{p-1} u,
$$

where the relaxation function $k$ is monotone decreasing with $k(+\infty)=1, m \geq 1,1 \leq$ $p<6$. When the source is stronger than dissipations, i.e. $p>\max \{m, \sqrt{k(0)}\}$, they obtained some finite time blow-up results with positive initial energy. In particular, they obtained the existence of certain solutions which blow up in finite time for initial data at arbitrary energy level. In [17, the abstract thermo-elastic system is considered,

$$
\left\{\begin{array}{l}
u^{\prime \prime}+A u+B u^{\prime}-\int_{0}^{\infty} g(s) u_{x x}(t-s) d s-A^{\alpha} \theta=0 \\
\theta^{\prime}+k A^{\beta} \theta \theta+A^{\alpha} u^{\prime}=0 \\
u(-t)=u_{0}(t), \quad u^{\prime}(0)=u_{1}, \theta(0)=\theta_{0}
\end{array}\right.
$$

in which $u$ is the displacement vector, $\theta$ is the temperature difference, and $\alpha \in$ $[0,1), \beta \in(0,1]$ are constants. $H$ is a real Hilbert space equipped with the inner product $\langle.,$.$\rangle and the related norm \|$.$\| . The operators A: D(A) \rightarrow H$ and $B: D(B) \rightarrow H$ are self-adjoint linear positive definite operators. Under suitable conditions on the order of the coupling, the memory kernel function and the initial values, the well-posedness and the general decay rate of solution is given by semigroup theory and perturbed energy functional technique to allow a wider thermo-elastic systems.

Without infinite memory term, when $\lambda=0$ in (1.1), Triet and his collaborator in [30] considered an IBVP for a nonlinear Kirchhoff-Love equation

$$
\begin{gathered}
u_{t t}-\frac{\partial}{\partial x}\left[B\left(x, t, u,\|u\|^{2},\left\|u_{x}\right\|^{2},\left\|u_{t}\right\|^{2},\left\|u_{x t}\right\|^{2}\right)\left(u_{x}+\lambda_{1} u_{x t}+u_{x t t}\right)\right]+\lambda u_{t} \\
=F\left(x, t, u, u_{x}, u_{t}, u_{x t},\|u(t)\|^{2},\left\|u_{x}(t)\right\|^{2},\left\|u_{t}(t)\right\|^{2},\left\|u_{x t}(t)\right\|^{2}\right) \\
-\frac{\partial}{\partial x}\left[G\left(x, t, u, u_{x}, u_{t}, u_{x t},\|u(t)\|^{2},\left\|u_{x}(t)\right\|^{2},\left\|u_{t}(t)\right\|^{2},\left\|u_{x t}(t)\right\|^{2}\right)\right] \\
+f(x, t), \quad x \in \Omega=(0,1), 0<t<T, \\
u(0, t)=u(1, t)=0, \\
u(x, 0)=\tilde{u}_{0}(x), \quad u_{t}(x, 0)=\tilde{u}_{1}(x),
\end{gathered}
$$

where $\lambda>0, \lambda_{1}>0$ are constants and $\tilde{u}_{0}, \tilde{u}_{1} \in H_{0}^{1} \cap H^{2} ; f, F$ and $G$ are given functions. By applying the Faedo-Galerkin, the authors proved existence and uniqueness of a solution and by constructing Lyapunov functional, they proved a blow-up of the solution with a negative initial energy, and established a sufficient condition for the exponential decay of weak solutions. This last results extend their previous 
results in 29 .

Remark 1.1. We should mention here that the presence of the term $-(F[u])_{x}$ can ensure only the local existence (can't be continue to be global in time), $i$. e., if we take $-(F[u])_{x} \equiv 0$, under the same condition, (Hyp1)-(Hyp 4), 3.4) and 3.10, we arrive to the blow up for all $t>0$. That is the term $F[u]$ is strong enough to prevent the existence of solutions.

This paper is organized as follows: In the second section, because of the nonlinearities, we combine a three techniques to prove the local existence of unique weak solution in Theorem 2.3. In the third section, the blow up results with negative initial energy is obtained in Theorem 3.3. under certain conditions on the sources and the function $g$. It is not surprising that this work is inspired from [22, 223, 30, 33 .

\section{EXISTENCE OF A WEAK SOLUTION}

The weak formulation. We define in the following, the weak solution to of (1.1)(1.4.

Definition 2.1. A function $u$ is said to be a weak solution of (1.1)-1.4 on $[0, T]$ if

$$
u, u^{\prime}, u^{\prime \prime} \in L^{\infty}\left(0, T ; H_{0}^{1} \cap H^{2}\right),
$$

such that $u$ satisfies the variational equation

$$
\begin{aligned}
& \int_{0}^{1} u^{\prime \prime} w d x+\int_{0}^{1}\left(\lambda_{0} u_{x}+\lambda_{1} u_{x}^{\prime}+u_{x}^{\prime \prime}\right) w_{x} d x \\
& -\lambda \int_{0}^{1} \int_{0}^{\infty} g(s) u_{x}(t-s) d s w_{x} d x \\
& =\int_{0}^{1} f w d x+\int_{0}^{1} F[u] w d x+\int_{0}^{1} F[u] w_{x} d x
\end{aligned}
$$

for all test function $w \in H_{0}^{1}$, for almost all $t \in(0, T)$.

The following famous and widely used technical lemma will play an important role in the sequel.

Lemma 2.2. For any $v \in C^{1}\left(0, T, H_{0}^{1}\right)$ we have

$$
\begin{aligned}
& \int_{0}^{1} \int_{0}^{\infty} g(s) v_{x x}(t-s) v^{\prime}(t) d s d x \\
= & \frac{1}{2} \frac{d}{d t} \int_{0}^{\infty} g(s) \int_{0}^{1}|v(t-s)-v(t)|^{2} d x d s-\frac{1}{2} \frac{d}{d t} \int_{0}^{\infty} g(s) d s \int_{0}^{1}\left|v_{x}(t)\right|^{2} d x \\
& -\frac{1}{2} \int_{0}^{\infty} g^{\prime}(s) \int_{0}^{1}|v(t-s)-v(t)|^{2} d x d s .
\end{aligned}
$$

Proof. It's not hard to see

$$
\int_{0}^{1} \int_{0}^{\infty} g(s) v_{x x}(t-s) v^{\prime}(t) d s d x
$$




$$
\begin{aligned}
= & -\int_{0}^{\infty} g(s) \int_{0}^{1} v_{x}^{\prime}(t) v_{x}(t-s) d x d s \\
= & -\int_{0}^{\infty} g(s) \int_{0}^{1} v_{x}^{\prime}(t)\left[v_{x}(t-s)-v_{x}(t)\right] d x d s \\
& -\int_{0}^{\infty} g(s) \int_{0}^{1} v_{x}^{\prime}(t) v_{x}(t) d x d s .
\end{aligned}
$$

Consequently,

$$
\begin{aligned}
& \int_{0}^{1} \int_{0}^{\infty} g(s) v_{x x}(s) v^{\prime}(t) d s d x \\
= & \frac{1}{2} \int_{0}^{\infty} g(s) \frac{d}{d t} \int_{0}^{1}\left|v_{x}(t-s)-v_{x}(t)\right|^{2} d x d s \\
& -\int_{0}^{\infty} g(s)\left(\frac{d}{d t} \frac{1}{2} \int_{0}^{1}\left|v_{x}(t)\right|^{2} d x\right) d s,
\end{aligned}
$$

which implies,

$$
\begin{aligned}
& \int_{0}^{1} \int_{0}^{\infty} g(s) v_{x x}(s) v^{\prime}(t) d s d x \\
= & \frac{1}{2} \frac{d}{d t}\left[\int_{0}^{\infty} g(s) \int_{0}^{1}\left|v_{x}(t-s)-v_{x}(t)\right|^{2} d x d s\right] \\
& -\frac{1}{2} \frac{d}{d t}\left[\int_{0}^{\infty} g(s) d s \int_{0}^{1}\left|v_{x}(t)\right|^{2} d x\right] \\
& -\frac{1}{2} \int_{0}^{\infty} g^{\prime}(s) \int_{0}^{1}\left|v_{x}(t-s)-v_{x}(t)\right|^{2} d x d s,
\end{aligned}
$$

First main theorem. Various existence and uniqueness, as well as Faedo-Galerkin method, have been obtained in the lase decades for nonlinear IBVPs in Sobolev spaces (see 7], 12, 16, [14, ...). Now, we consider the existence of a local solution for 1.1-1.4, with $\lambda \in \mathbb{R}, \lambda_{0}, \lambda_{1}>0$.

Theorem 2.3. Let $u_{0}(0), u_{1} \in H_{0}^{1} \cap H^{2}$ be given. Assume that (Hyp1)-(Hyp4) hold. Then Problem (1.1) - 1.4) has a unique local solution $u$ and

$$
u, u^{\prime}, u^{\prime \prime} \in L^{\infty}\left(0, T_{*} ; H_{0}^{1} \cap H^{2}\right),
$$

for some $T_{*}>0$ small enough.

Proof of Theorem 2.3. In the first step of this proof, using linearization method for a nonlinear term to construct a linear recurrent sequence $\left\{u_{m}\right\}$. Then, the FaedoGalerkin method combined with the weak compactness method shows that $\left\{u_{m}\right\}$ converges to $u$ which is exactly a unique local solution of (1.1) -1.4 .

Step 1. Let $T>0$ be fixed, let $M>0$, we put

$$
\begin{gathered}
K_{M}(f)=\sqrt{\|f\|_{L^{2}((0,1) \times(0, T))}^{2}+\left\|f^{\prime}\right\|_{L^{2}((0,1) \times(0, T))}^{2}+\left\|f_{x}\right\|_{L^{2}((0,1) \times(0, T))}^{2}}, \\
\|F\|_{C^{0}\left([0,1] \times[0, T] \times[-M, M]^{4}\right)}=\sup _{\left(x, t, y_{1}, \ldots, y_{4}\right) \in[0,1] \times[0, T] \times[-M, M]^{4}}\left|F\left(x, t, y_{1}, \ldots, y_{4}\right)\right|,
\end{gathered}
$$




$$
\begin{aligned}
\bar{F}_{M} & =\|F\|_{C^{1}\left([0,1] \times[0, T] \times[-M, M]^{4}\right)} \\
& =\|F\|_{C^{0}\left([0,1] \times[0, T] \times[-M, M]^{4}\right)} \\
& +\sum_{i=1}^{6}\left\|D_{i} F\right\|_{C^{0}\left([0,1] \times[0, T] \times[-M, M]^{4}\right)} .
\end{aligned}
$$

For some $T_{*} \in(0, T]$ and $M>0$, we put

$$
\begin{gathered}
W\left(M, T_{*}\right)=\left\{v, v^{\prime} \in L^{\infty}\left(0, T_{*} ; H_{0}^{1} \cap H^{2}\right): v^{\prime \prime} \in L^{\infty}\left(0, T_{*} ; H_{0}^{1}\right),\right. \\
\text { with }\|v\|_{L^{\infty}\left(0, T_{*} ; H_{0}^{1} \cap H^{2}\right)}, \\
\left.\left\|v^{\prime}\right\|_{L^{\infty}\left(0, T_{*} ; H_{0}^{1} \cap H^{2}\right)},\left\|v^{\prime \prime}\right\|_{L^{\infty}\left(0, T_{*} ; H_{0}^{1}\right)} \leq M\right\} \\
W_{1}\left(M, T_{*}\right)=\left\{v \in W\left(M, T_{*}\right): v^{\prime \prime} \in L^{\infty}\left(0, T_{*} ; H_{0}^{1} \cap H^{2}\right)\right\} .
\end{gathered}
$$

We can now establish the linear recurrent sequence $\left\{u_{m}\right\}$ and choosing $u_{0}(t) \equiv 0$, suppose that

$$
u_{m-1} \in W_{1}\left(M, T_{*}\right),
$$

and associate with problem $(1.1)-(1.4)$ the following problem.

Using Lemma 2.2. find $u_{m} \in W_{1}\left(M, T_{*}\right)(m \geq 1)$ which satisfies

$$
\begin{aligned}
& \int_{0}^{1} u_{m}^{\prime \prime} w d x+\int_{0}^{1}\left(\lambda_{0} u_{x m}+\lambda_{1} u_{x m}^{\prime}+u_{x m}^{\prime \prime}\right) w_{x} d x \\
& -\lambda \int_{0}^{\infty} g(s) d s \int_{0}^{1} u_{x m} w_{x} d x-\lambda \int_{0}^{\infty} g(s) \int_{0}^{1}\left(u_{x m}(t-s)-u_{x m}\right) w_{x} d x d s \\
& =\int_{0}^{1} f w d x+\int_{0}^{1} F_{m}[u] w d x+\int_{0}^{1} F_{m}[u] w_{x} d x, \quad \forall w \in H_{0}^{1}, \\
& u_{m}(-t)=u_{0}(t), \quad u_{m}^{\prime}(0)=u_{1}, t \in[0, T]
\end{aligned}
$$

where

$$
\begin{aligned}
F_{m}[u] & =F\left[u_{m-1}\right] \\
& =F\left(x, t, u_{m-1}, u_{x m-1}, u_{m-1}^{\prime}, u_{x m-1}^{\prime}\right),
\end{aligned}
$$

Proposition 2.4. Let $u_{0}(0), u_{1} \in H_{0}^{1} \cap H^{2}$ be given, the first term of sequence $u_{0}(t) \equiv 0$. Assume that (Hyp1)-(Hyp4) hold. Then there exist positive constants $M, T_{*}>0$ such that, there exists a recurrent sequence $\left\{u_{m}\right\} \subset W_{1}\left(M, T_{*}\right)$ defined by $2.5-2.7$.

Proof of Proposition 2.4. We use the standard Faedo-Galerkin method to prove our result. Consider a special orthonormal basis $\left\{w_{j}\right\}_{j=1}^{\infty}$ on $H_{0}^{1}$, formed by the eigenfunctions of the operator $-\frac{\partial^{2}}{\partial x^{2}}$.

Let $V_{k}=\operatorname{span}\left\{w_{1}, w_{2}, \ldots, w_{k}\right\}$ and the projections of the history and initial data 
on the finite-dimensional subspace $V_{k}$ are given by

$$
\begin{aligned}
u_{0 k}(t) & =\sum_{j=1}^{k} \alpha_{j}^{(k)}(t) w_{j}, \\
u_{1 k} & =\sum_{j=1}^{k} \beta_{j}^{(k)} w_{j},
\end{aligned}
$$

where

$$
\begin{gathered}
\alpha_{j}^{(k)}(t)=\int_{0}^{1} u_{0}(t) w_{j} d x \\
\beta_{j}^{(k)}(t)=\int_{0}^{1} u_{1} w_{j} d x
\end{gathered}
$$

We seek $k$ functions $\varphi_{m j}^{(k)}(t) \in C^{2}[0, T], 1 \leq j \leq k$, such that the expression in form

$$
u_{m}^{(k)}=\sum_{j=1}^{k} \varphi_{m j}^{(k)} w_{j},
$$

solves the problem

$$
\begin{aligned}
& \int_{0}^{1} u_{m}^{\prime \prime}{ }^{(k)} w_{j} d x+\int_{0}^{1}\left(u_{x m}^{(k)}+\lambda_{1} u_{x m}^{\prime(k)}+u_{x m}^{\prime \prime}\right) w_{j x} d x \\
& -\lambda \int_{0}^{\infty} g(s) d s \int_{0}^{1} u_{x m}^{(k)} w_{j x} d x-\lambda \int_{0}^{\infty} g(s) \int_{0}^{1}\left(u_{x m}^{(k)}(t-s)-u_{x m}^{(k)}(s)\right) w_{j x} d x d s \\
& =\int_{0}^{1} f w_{j} d x+\int_{0}^{1} F_{m} w_{j} d x+\int_{0}^{1} F_{m} w_{j x} d x, \quad 1 \leq j \leq k, \\
& u_{m}^{(k)}(0)(-t)=u_{0 k}(t), \quad u_{m}^{\prime(k)}(0)=u_{1 k},
\end{aligned}
$$

in which

$$
\begin{aligned}
& u_{0 k} \rightarrow u_{0}(t) \quad \text { strongly in } H_{0}^{1} \cap H^{2}, \\
& u_{1 k}=\rightarrow u_{1} \quad \text { strongly in } H_{0}^{1} \cap H^{2} .
\end{aligned}
$$

This leads to a system of ODEs for unknown functions $\varphi_{m i}^{(k)}$. Based on standard existence theory for ODE, System 2.10 admits a unique solution $\varphi_{m j}^{(k)}, 1 \leq j \leq k$ on interval $[0, T]$, by $(2.5)$ and the argument in [5], the proof is completed.

A priori estimates. The next estimates prove that there exist positive constants $M, T_{*}>0$ such that $u_{m}^{(k)} \in W\left(M, T_{*}\right)$, for all $m$ and $k$. We partially estimate the terms of the associated energy. Multiplying the equation in 1.1 by $u_{m}^{\prime}(k)$ integrating over $[0,1]$ to get

$$
\begin{aligned}
& \int_{0}^{1} u_{m}^{\prime \prime(k)} u_{m}^{\prime(k)} d x+\int_{0}^{1}\left(\lambda_{0} u_{x m}^{(k)}+\lambda_{1} u_{x m}^{\prime(k)}+u_{x m}^{\prime \prime(k)}\right) u_{x m}^{\prime(k)} d x \\
& +\lambda \int_{0}^{1} \int_{0}^{\infty} g(s) u_{x m}^{(k)}(t-s) d s u_{x m}^{\prime(k)} d x \\
& =\int_{0}^{1} F\left[u_{m}^{(k)}\right] u_{m}^{\prime(k)} d x+\int_{0}^{1} F\left[u_{m}^{(k)}\right] u_{x m}^{\prime(k)} d x+\int_{0}^{1} f u_{m}^{\prime(k)} d x .
\end{aligned}
$$


Using results in Lemma 2.2, we obtain

$$
\begin{aligned}
& \frac{1}{2} \frac{d}{d t}\left[\int_{0}^{1}\left(\left|u_{m}^{\prime(k)}\right|^{2}+\left(\lambda_{0}-\lambda \int_{0}^{\infty} g(s) d s\right)\left|u_{x m}^{(k)}\right|^{2}+\left|u_{x m}^{\prime(k)}\right|^{2} d x\right) d x\right. \\
& \left.+\lambda \int_{0}^{\infty} g(s) \int_{0}^{1}\left|u_{x m}^{(k)}(t-s)-u_{x m}^{(k)}(t)\right|^{2} d x d s\right] \\
& +\lambda_{1} \int_{0}^{1}\left|u_{x m}^{\prime(k)}\right|^{2} d x-\lambda \int_{0}^{\infty} g^{\prime}(s) \int_{0}^{1}\left|u_{x m}^{(k)}(t-s)-u_{x m}^{(k)}(t)\right|^{2} d x d s \\
& =\int_{0}^{1} F\left[u_{m}^{(k)}\right] u_{m}^{\prime(k)} d x+\int_{0}^{1} F\left[u_{m}^{(k)}\right] u_{x m}^{\prime(k)} d x+\int_{0}^{1} f_{m} u_{m}^{(k)} d x .
\end{aligned}
$$

Let us denote the LHS of $(2.12)$ as

$$
\begin{aligned}
e^{(k)}\left(u_{m}\right) & =\int_{0}^{1}\left[\left|u_{m}^{\prime}(k)\right|^{2}+\left(\lambda_{0}-\lambda \int_{0}^{\infty} g(s) d s\right)\left|u_{x m}^{(k)}\right|^{2}+\left|u_{x m}^{\prime(k)}\right|^{2} d x\right] d x \\
& +\lambda \int_{0}^{\infty} g(s) \int_{0}^{1}\left|u_{x m}^{(k)}(t-s)-u_{x m}^{(k)}(t)\right|^{2} d x d s \\
& +2 \lambda_{1} \int_{0}^{t} \int_{0}^{1}\left|u_{x m}^{\prime}(k)\right|^{2} d x d s-\lambda \int_{0}^{t} \int_{0}^{\infty} g^{\prime}(s) \int_{0}^{1}\left|u_{x m}^{(k)}(t-s)-u_{x m}^{(k)}(t)\right|^{2} d x d s d \tau
\end{aligned}
$$

and

$$
\begin{aligned}
e^{(k)}\left(u_{x m}\right) & =\int_{0}^{1}\left[\left|u_{x m}^{\prime(k)}\right|^{2}+\left(\lambda_{0}-\lambda \int_{0}^{\infty} g(s) d s\right)\left|u_{x x m}^{(k)}\right|^{2}+\left|u_{x x m}^{\prime(k)}\right|^{2} d x\right] d x \\
& +\lambda \int_{0}^{\infty} g(s) \int_{0}^{1}\left|u_{x x m}^{(k)}(t-s)-u_{x x m}^{(k)}(t)\right|^{2} d x d s \\
& +2 \lambda_{1} \int_{0}^{t} \int_{0}^{1}\left|u_{x x m}^{\prime(k)}\right|^{2} d x d s-\lambda \int_{0}^{t} \int_{0}^{\infty} g^{\prime}(s) \int_{0}^{1}\left|u_{x x m}^{(k)}(t-s)-u_{x x m}^{(k)}(t)\right|^{2} d x d s \tau,
\end{aligned}
$$

and

$$
\begin{aligned}
e^{(k)}\left(u_{m}^{\prime}\right) & =\int_{0}^{1}\left[\left|u_{m}^{\prime \prime}(k)\right|^{2}+\left(\lambda_{0}-\lambda \int_{0}^{\infty} g(s) d s\right)\left|u_{x m}^{\prime(k)}\right|^{2}+\left|u_{x m}^{\prime \prime(k)}\right|^{2} d x\right] d x \\
& +\lambda \int_{0}^{\infty} g(s) \int_{0}^{1}\left|u_{x m}^{\prime(k)}(t-s)-u_{x m}^{\prime(k)}(t)\right|^{2} d x d s \\
& +2 \lambda_{1} \int_{0}^{t} \int_{0}^{1}\left|u_{x m}^{\prime \prime(k)}\right|^{2} d x d s-\lambda \int_{0}^{t} \int_{0}^{\infty} g^{\prime}(s) \int_{0}^{1}\left|u_{x m}^{\prime(k)}(t-s)-u_{x m}^{\prime(k)}(t)\right|^{2} d x d s d \tau .
\end{aligned}
$$

Put

$$
E_{m}^{(k)}(t)=e^{(k)}\left(u_{m}\right)+e^{(k)}\left(u_{x m}\right)+e^{(k)}\left(u_{m}^{\prime}\right) .
$$


Then

$$
\begin{aligned}
& E_{m}^{(k)}(t) \\
& =E_{m}^{(k)}(0)+2 \int_{0}^{t} \int_{0}^{1} f(s) u_{m}^{\prime(k)}(s) d x d s+2 \int_{0}^{t} \int_{0}^{1} f_{x}(s) u_{x m}^{\prime(k)}(s) d x d s \\
& \quad+2 \int_{0}^{t} \int_{0}^{1} f^{\prime}(s) u_{m}^{\prime \prime}(k)(s) d x d s+2 \int_{0}^{t} \int_{0}^{1} F_{m}(s) u_{x m}^{\prime(k)}(s) d x d s \\
& +2 \int_{0}^{t} \int_{0}^{1} F_{m}(s) u_{m}^{\prime(k)}(s) d x d s+2 \int_{0}^{t} \int_{0}^{1} F_{x m}(s) u_{x x m}^{\prime(k)}(s) d x d s \\
& +2 \int_{0}^{t} \int_{0}^{1} F_{m}^{\prime}(s) u_{m}^{\prime \prime}(k)(s) d x d s+2 \int_{0}^{1} \int_{0}^{\prime} F_{m}^{\prime}(s) u_{x m}^{\prime \prime}(k)(s) d x d s \\
& +2 \int_{0}^{t} \int_{0}^{1} F_{x m}(s) u_{x m}^{\prime(k)}(s) d x d s .
\end{aligned}
$$

We need, now, to estimate

$$
A_{m}^{(k)}=\int_{0}^{1}\left|u_{m}^{\prime \prime(k)}(0)\right|^{2} d x+\int_{0}^{1}\left|u_{x m}^{\prime \prime(k)}(0)\right|^{2} d x .
$$

Let $w_{j}=u_{m}^{\prime \prime}{ }^{(k)}$ in 2.10 and integrate by parts, taking $t \rightarrow 0_{+}$in the first term, to obtain

$$
\begin{aligned}
& \int_{0}^{1}\left|u_{m}^{\prime \prime(k)}(0)\right|^{2} d x+\int_{0}^{1}\left|u_{x m}^{\prime \prime(k)}(0)\right|^{2} d x \\
& +\int_{0}^{1}\left(\left[\lambda_{0}-\lambda \int_{0}^{\infty} g(s) d s\right] u_{0 k x}+\lambda_{1} u_{1 k x}\right) u_{x m}^{\prime \prime(k)}(0) d x \\
& +\lambda \int_{0}^{\infty} g(s) \int_{0}^{1}\left(u_{0 k x}(0)-u_{0 k x}(-s)\right) u_{m}^{\prime \prime(k)}(0) d x d s \\
& =\int_{0}^{1} f(0) u_{m}^{\prime \prime(k)}(0) d x+\int_{0}^{1} F_{m}(0) u_{m}^{\prime \prime(k)}(0) d x+\int_{0}^{1} F_{m}(0) u_{x m}^{\prime \prime(k)}(0) d x .
\end{aligned}
$$

Then

$$
\begin{aligned}
A_{m}^{(k)} & \leq \int_{0}^{1}\left(\left[\lambda_{0}-\lambda \int_{0}^{\infty} g(s) d s\right] u_{0 k x}+\lambda_{1} u_{1 k x}+F_{m}(0)\right) u_{x m}^{\prime \prime(k)}(0) d x \\
& +\lambda \int_{0}^{\infty} g(s) \int_{0}^{1}\left(u_{0 k x}(0)-u_{0 k x}(-s)\right) u_{m}^{\prime \prime}(k)(0) d x d s \\
& +\int_{0}^{1} f(0) u_{m}^{\prime \prime}(k)(0) d x+\int_{0}^{1} F_{m}(0) u_{m}^{\prime \prime}(k)(0) d x \\
& \leq \int_{0}^{1}\left[\left[\lambda_{0}-\lambda \int_{0}^{\infty} g(s) d s\right] u_{0 k x}+\lambda_{1} u_{1 k x}+2 F_{m}(0)+f(0)\right. \\
& \left.+\lambda \int_{0}^{\infty} g(s)\left(u_{0 k x}(0)-u_{0 k x}(-s)\right)\right] d x\left[A_{m}^{(k)}\right]^{1 / 2} \\
\leq & {\left[\int _ { 0 } ^ { 1 } \left[\left[\lambda_{0}-\lambda \int_{0}^{\infty} g(s) d s\right] u_{0 k x}+\lambda_{1} u_{1 k x}+2 F_{m}(0)+f(0)\right.\right.}
\end{aligned}
$$




$$
\begin{aligned}
& \left.\left.+\lambda \int_{0}^{\infty} g(s)\left(u_{0 k x}(0)-u_{0 k x}(-s)\right)\right] d x\right]^{2} \\
& \leq \xi, \quad \text { for all } m, k,
\end{aligned}
$$

because, $\int_{0}^{1}\left|F_{m}(0)\right| d x$ is a constant independent of $m$, where $\xi$ is a constant depending only on $f, u_{0}, u_{1}, F, \lambda_{0}, \lambda, \lambda_{1}$ and $\int_{0}^{\infty} g(s) d s$. Equations 2.11, 2.13) and (2.15) imply that

$$
\begin{aligned}
E_{m}^{(k)}(0) & =\int_{0}^{1}\left[\left|u_{1 k}\right|^{2}+\left(\lambda_{0}-\lambda \int_{0}^{\infty} g(s) d s\right)\left|u_{0 k x}\right|^{2}+\left|u_{1 k x}\right|^{2} d x\right] d x \\
& +\lambda \int_{0}^{\infty} g(s) \int_{0}^{1}\left|u_{0 k x}(-s)-u_{0 k x}(0)\right|^{2} d x d s \\
& +\int_{0}^{1}\left[\left|u_{1 k x}\right|^{2}+\left(\lambda_{0}-\lambda \int_{0}^{\infty} g(s) d s\right)\left|u_{0 k x x}\right|^{2}+\left|u_{1 k x x}\right|^{2} d x\right] d x \\
& +\lambda \int_{0}^{\infty} g(s) \int_{0}^{1}\left|u_{0 k x x}(-s)-u_{0 k x x}(0)\right|^{2} d x d s \\
& +A_{m}^{(k)}+\int_{0}^{1}\left(\lambda_{0}-\lambda \int_{0}^{\infty} g(s) d s\right)\left|u_{1 k x}\right|^{2} d x \\
& +\lambda \int_{0}^{\infty} g(s) \int_{0}^{1}\left|u_{1 k x}-u_{1 k x}\right|^{2} d x d s \\
\leq & \xi_{0}, \quad \text { for all } m, k \in \mathbb{N}
\end{aligned}
$$

where $\xi_{0}$ is also a constant depending only on $f, u_{0}, u_{1}, F, \lambda_{0}, \lambda, \lambda_{1}$ and $\int_{0}^{\infty} g(s) d s$. We then now estimate the other terms of 2.14). By the Cauchy - Schwartz inequality, we obtain

$$
\begin{aligned}
& E_{m}^{(k)}(t) \leq \xi_{0} \\
& +\|f\|_{L^{2}((0,1) \times(0, T))}^{2}+\int_{0}^{t} \int_{0}^{1}\left|u_{m}^{\prime(k)}\right|^{2} d s d x ; \\
& +\left\|f_{x}\right\|_{L^{2}((0,1) \times(0, T))}^{2}+\int_{0}^{t} \int_{0}^{1}\left|u_{x m}^{\prime(k)}\right|^{2} d x d s ; \\
& +\left\|f^{\prime}\right\|_{L^{2}((0,1) \times(0, T))}^{2}+\int_{0}^{t} \int_{0}^{1}\left|u_{m}^{\prime \prime(k)}\right|^{2} d x d s ; \\
& +2 \int_{0}^{t} \int_{0}^{1} F_{m}(s) u_{x m}^{\prime(k)}(s) d x d s \\
& +2 \int_{0}^{t} \int_{0}^{1} F_{m}(s) u_{m}^{\prime(k)}(s) d x d s+2 \int_{0}^{t} \int_{0}^{1} F_{x m}(s) u_{x x m}^{\prime(k)}(s) d x d s \\
& +2 \int_{0}^{t} \int_{0}^{1} F_{m}^{\prime}(s) u_{m}^{\prime \prime(k)}(s) d x d s+2 \int_{0}^{t} \int_{0}^{1} F_{m}^{\prime}(s) u_{x m}^{\prime \prime(k)}(s) d x d s \\
& +2 \int_{0}^{t} \int_{0}^{1} F_{x m}(s) u_{x m}^{\prime(k)}(s) d x d s .
\end{aligned}
$$


By (Hyp1), 2.13, we have

$$
\begin{aligned}
& E_{m}^{(k)}(t) \leq \xi_{0}+\|f\|_{H^{1}((0,1) \times(0, T))}^{2}+c \int_{0}^{t} E_{m}^{(k)}(s) d s \\
& +2 \int_{0}^{t} \int_{0}^{1} F_{m}(s) u_{x m}^{\prime(k)}(s) d x d s \\
& +2 \int_{0}^{t} \int_{0}^{1} F_{m}(s) u_{m}^{\prime(k)}(s) d x d s+2 \int_{0}^{t} \int_{0}^{1} F_{x m}(s) u_{x x m}^{\prime(k)}(s) d x d s \\
& +2 \int_{0}^{t} \int_{0}^{1} F_{m}^{\prime}(s) u_{m}^{\prime \prime(k)}(s) d x d s+2 \int_{0}^{t} \int_{0}^{1} F_{m}^{\prime}(s) u_{x m}^{\prime \prime(k)}(s) d x d s \\
& +2 \int_{0}^{t} \int_{0}^{1} F_{x m}(s) u_{x m}^{\prime(k)}(s) d x d s .
\end{aligned}
$$

We have

$$
\begin{aligned}
& E_{m}^{(k)}(t) \leq \xi_{0}+\|f\|_{H^{1}((0,1) \times(0, T))}^{2}+c \int_{0}^{t} E_{m}^{(k)}(s) d s \\
& +T_{*} \bar{F}_{M}^{2}+\int_{0}^{t} \int_{0}^{1}\left|u_{x m}^{\prime(k)}(s)\right|^{2} d x d s+T_{*} \bar{F}_{M}^{2}+\int_{0}^{t} \int_{0}^{1}\left|u_{m}^{\prime(k)}\right|^{2} d x d s \\
& +2 \int_{0}^{t} \int_{0}^{1} F_{x m}(s) u_{x x m}^{\prime(k)}(s) d x d s+T_{*} \bar{F}_{M}^{2}+\int_{0}^{t} \int_{0}^{1}\left|u_{x m}^{\prime(k)}(s)\right|^{2} d x d s . \\
& +2 \int_{0}^{t} \int_{0}^{1} F_{m}^{\prime}(s) u_{m}^{\prime \prime(k)}(s) d x d s+2 \int_{0}^{t} \int_{0}^{1} F_{m}^{\prime}(s) u_{x m}^{\prime \prime(k)}(s) d x d s
\end{aligned}
$$

By (Hyp1), 2.13 and remarking from 1.2 that

$$
\begin{aligned}
F_{x m}^{\prime}(t)= & D_{1} F\left[u_{m-1}\right]+D_{3} F\left[u_{m-1}\right] u_{x m-1}^{\prime}+D_{4} F\left[u_{m-1}\right] u_{x x m-1}^{\prime} \\
& +D_{5} F\left[u_{m-1}\right] u_{x m-1}^{\prime}+D_{6} F\left[u_{m-1}\right] u_{x x m-1}^{\prime} .
\end{aligned}
$$

Then

$$
\begin{aligned}
& E_{m}^{(k)}(t) \leq \xi_{0}+\|f\|_{H^{1}((0,1) \times(0, T))}^{2} \\
& +2 T_{*}\left[1+2(1+4 M)^{2}\right] \bar{F}_{M}^{2}+c \int_{0}^{t} E_{m}^{(k)}(s) d s .
\end{aligned}
$$

We choose $M>0$ sufficiently large such that

$$
E_{0}+\|f\|_{H^{1}((0,1) \times(0, T))}^{2} \leq \frac{1}{2} M^{2},
$$

and then choose $T_{*} \in(0, T]$ small enough such that

$$
\left(\frac{1}{2} M^{2}+T_{*}\left[1+2(1+4 M)^{2}\right] \bar{F}_{M}^{2}\right) \exp \left[2 T_{*}\right] \leq M^{2},
$$

and

$$
k_{T_{*}}=2 \sqrt{\bar{F}_{M}^{2}} \sqrt{T_{*} \exp \left[T_{*}\right]}<1,
$$

Then

$$
u_{m}^{(k)} \in W\left(M, T_{*}\right) \text {, for all } m \text { and } k .
$$


Pass to the limit.

By 2.22, there exists a subsequence of $\left\{u_{m}^{(k)}\right\}$, such that

$$
\begin{gathered}
u_{m}^{(k)} \rightarrow u_{m} \quad \text { in } L^{\infty}\left(0, T_{*} ; H_{0}^{1} \cap H^{2}\right) \text { weakly*, } \\
u_{m}^{\prime(k)} \rightarrow u_{m}^{\prime} \quad \text { in } L^{\infty}\left(0, T_{*} ; H_{0}^{1} \cap H^{2}\right) \text { weakly*, } \\
u_{m}^{\prime \prime(k)} \rightarrow u_{m}^{\prime \prime} \quad \text { in } L^{\infty}\left(0, T_{*} ; H_{0}^{1}\right) \text { weakly*, } \\
u_{m} \in W\left(M, T_{*}\right) .
\end{gathered}
$$

Passing to limit in (2.10), 2.11), it is clear to see that $u_{m}$ is satisfying (2.6), 2.7) in $L^{2}\left(0, T_{*}\right)$. Furthermore, $(2.6)_{1}$ and 2.25$)_{4}$ imply that

$$
\begin{aligned}
& \left(\lambda_{0} u_{m}+\lambda_{1} u_{m}^{\prime}+u_{m}^{\prime \prime}+\lambda \int_{0}^{\infty} g(s) u_{m}(t-s) d s\right) x x \\
& =u_{m}^{\prime \prime}-F\left[u_{m}\right]-\left(F\left[u_{m}\right]\right)_{x}-f \\
& \equiv \Psi_{m} \in L^{\infty}\left(0, T_{*} ; H_{0}^{1} \cap H^{2}\right) .
\end{aligned}
$$

We deduce that, if $u_{m} \in L^{\infty}\left(0, T_{*} ; H_{0}^{1} \cap H^{2}\right)$, then $u_{m}^{\prime}, u_{m}^{\prime \prime} \in L^{\infty}\left(0, T_{*} ; H_{0}^{1} \cap H^{2}\right)$. So we obtain $u_{m} \in W_{1}\left(M, T_{*}\right)$. This completes the proof of Proposition 2.4.

Step 2. Let the Banach space

$$
W_{1}\left(T_{*}\right)=\left\{v \in L^{\infty}\left(0, T_{*} ; H_{0}^{1}\right): v^{\prime} \in L^{\infty}\left(0, T_{*} ; H_{0}^{1}\right)\right\},
$$

with respect to the norm

$$
\|v\|_{W_{1}\left(T_{*}\right)}=\|v\|_{L^{\infty}\left(0, T_{*} ; H_{0}^{1}\right)}+\left\|v^{\prime}\right\|_{L^{\infty}\left(0, T_{*} ; H_{0}^{1}\right)} .
$$

We will show the convergence of $\left\{u_{m}\right\}$ to the solution of our problem in the next Lemma.

Lemma 2.5. Let (Hyp1)-(Hyp4) hold. Then

(i) Problem (1.1)-(1.4) has a unique weak solution $u \in W_{1}\left(M, T_{*}\right)$, where $M>$ 0 and $T_{*}>0$ are chosen constants as in Proposition 2.4.

(ii) The linear recurrent sequence $\left\{u_{m}\right\}$ defined by 2.5)-(2.7) converges to the solution $u$ of (1.1) - 1.4 strongly in the space $W_{1}\left(T_{*}\right)$

Proof. We use the result obtained in Proposition 2.4 and the compact embedding theorems.

Existence. We proved that $\left\{u_{m}\right\}$ is a Cauchy sequence in $W_{1}\left(T_{*}\right)$. In order to do this, let $w_{m}=u_{m+1}-u_{m}$. Then $w_{m}$ satisfies

$$
\begin{aligned}
& \int_{0}^{1} w_{m}^{\prime \prime} w d x+\int_{0}^{1}\left(\lambda_{0} w_{x m}+\lambda_{1} w_{x m}^{\prime}+w_{x m}^{\prime \prime}\right) w_{x} d x \\
& -\lambda \int_{0}^{1} \int_{0}^{\infty} g(s) w_{x m}(t-s) d s w_{x} d x \\
& =\int_{0}^{1}\left(F\left[w_{m+1}\right]-F\left[w_{m}\right]\right) w d x+\int_{0}^{1}\left(F\left[w_{m+1}\right]-F\left[w_{m}\right]\right) w_{x} d x \\
& w_{m}(0)=w_{m}^{\prime}(0)=0
\end{aligned}
$$


Consider (2.28) with $w=w_{m}^{\prime}$, and then integrating in $t$, we obtain by results in Lemma 2.2

$$
\begin{aligned}
& \int_{0}^{1}\left[\left|w_{m}^{\prime}\right|^{2}+\left(\lambda_{0}-\lambda \int_{0}^{\infty} g(s) d s\right)\left|w_{x m}\right|^{2}+\left|w_{x m}^{\prime}\right|^{2}\right] d x \\
& +\lambda \int_{0}^{\infty} g(s) \int_{0}^{1}\left|w_{x m}(t-s)-w_{x m}(t)\right|^{2} d x d s \\
& +2 \lambda_{1} \int_{0}^{t} \int_{0}^{1}\left|w_{x m}^{\prime}\right|^{2} d x d s-\lambda \int_{0}^{t} \int_{0}^{\infty} g^{\prime}(s) \int_{0}^{1}\left|w_{x m}(t-s)-w_{x m}(t)\right|^{2} d x d s d \tau \\
& =2 \int_{0}^{t} \int_{0}^{1}\left(F_{m+1}(s)-F_{m}(s)\right) w_{m}^{\prime}(s) d x d s \\
& +2 \int_{0}^{t} \int_{0}^{1}\left(F_{m+1}(s)-F_{m}(s)\right) w_{x m}^{\prime}(s) d x d s .
\end{aligned}
$$

By (Hyp2)-(Hyp4), 2.3), 2.5) and (2.25) we have

$$
\int_{0}^{1}\left|F_{m+1}(s)-F_{m}(s)\right|^{2} d x \leq 2 \bar{F}_{M} \int_{0}^{1}\left|w_{m-1}\right|^{2} d x,
$$

then

$$
\begin{aligned}
E_{m}(t) & \leq 2 \bar{F}_{M} \int_{0}^{1}\left|w_{m-1}\right|^{2} d x+\int_{0}^{t} \int_{0}^{1}\left|w_{m}^{\prime}\right|^{2} d x d s \\
& +2 \bar{F}_{M} \int_{0}^{1}\left|w_{m-1}\right|^{2} d x+\int_{0}^{t} \int_{0}^{1}\left|w_{x m}^{\prime}\right|^{2} d x d s \\
& \leq 4 \bar{F}_{M} \int_{0}^{1}\left|w_{m-1}\right|^{2} d x T_{*}+\int_{0}^{t} E_{m}(s) d s
\end{aligned}
$$

where

$$
\begin{aligned}
& E_{m}(t)=\int_{0}^{1}\left[\left|w_{m}^{\prime}\right|^{2}+\left(\lambda_{0}-\lambda \int_{0}^{\infty} g(s) d s\right)\left|w_{x m}\right|^{2}+\left|w_{x m}^{\prime}\right|^{2}\right] d x \\
& +\lambda \int_{0}^{\infty} g(s) \int_{0}^{1}\left|w_{x m}(t-s)-w_{x m}(t)\right|^{2} d x d s \\
& +2 \lambda_{1} \int_{0}^{t} \int_{0}^{1}\left|w_{x m}^{\prime}\right|^{2} d x d s-\lambda \int_{0}^{t} \int_{0}^{\infty} g^{\prime}(s) \int_{0}^{1}\left|w_{x m}(t-s)-w_{x m}(t)\right|^{2} d x d s d \tau .
\end{aligned}
$$

Thanks to Gronwall's Lemma, 2.30, we get

$$
\int_{0}^{1}\left|w_{m}\right| d x \leq k_{T_{*}} \int_{0}^{1}\left|w_{m-1}\right| d x \quad \forall m \in \mathbb{N},
$$

so

$$
\int_{0}^{1}\left|u_{m}-u_{m+p}\right| d x \leq M\left(1-k_{T_{*}}\right)^{-1} k_{T_{*}}^{m}, \quad \forall m, p \in \mathbb{N} .
$$

It follows that $\left\{u_{m}\right\}$ is a Cauchy sequence in $W_{1}\left(T_{*}\right)$, so there exists $u \in W_{1}\left(T_{*}\right)$ such that

$$
u_{m} \rightarrow u \text { strongly in } W_{1}\left(T_{*}\right) .
$$


Note that $u_{m} \in W_{1}\left(M, T_{*}\right)$, so there exists a subsequence $\left\{u_{m_{j}}\right\}$ of $\left\{u_{m}\right\}$ such that

$$
\begin{gathered}
u_{m_{j}} \rightarrow u \quad \text { in } L^{\infty}\left(0, T_{*} ; H_{0}^{1} \cap H^{2}\right) \text { weakly*, } \\
u_{m_{j}}^{\prime} \rightarrow u^{\prime} \quad \text { in } L^{\infty}\left(0, T_{*} ; H_{0}^{1} \cap H^{2}\right) \text { weakly*, } \\
u_{m_{j}}^{\prime \prime} \rightarrow u^{\prime \prime} \quad \text { in } L^{\infty}\left(0, T_{*} ; H_{0}^{1}\right) \text { weakly*, } \\
u \in W\left(M, T_{*}\right) .
\end{gathered}
$$

By (2.3), 2.5, 2.7) and 2.34 4 , we obtain

$$
\int_{0}^{1}\left|F_{m}(t)-F[u](t)\right| d x \leq 2(1+2 M) \bar{F}_{M} \int_{0}^{1}\left|u_{m-1}-u\right| d x
$$

Then $(2.33)$ and 2.35 imply

$$
F_{m} \rightarrow F[u] \text { strongly in } L^{\infty}\left(0, T_{*} ; L^{2}\right),
$$

Let us passing to limit in (2.6), 2.7) as $m=m_{j} \rightarrow \infty$, by (2.33), 2.34) and (2.36), there exists $u \in W\left(M, T_{*}\right)$ satisfying

$$
\begin{aligned}
& \int_{0}^{1} u^{\prime \prime} w d x+\int_{0}^{1}\left(\lambda_{0} u_{x}+\lambda_{1} u_{x}^{\prime}+u_{x}^{\prime \prime}\right) w_{x} d x \\
& -\lambda \int_{0}^{1} \int_{0}^{\infty} g(s) u_{x}(t-s) d s w_{x} d x \\
& =\int_{0}^{1} f w d x+\int_{0}^{1} F[u] w d x+\int_{0}^{1} F[u] w_{x} d x
\end{aligned}
$$

for all test function $w \in H_{0}^{1}$, for almost all $t \in(0, T)$ and satisfying the initial conditions.

Uniqueness. Let $u_{1}, u_{2}$ be two weak solutions of (1.1)-1.4 , such that

$$
u_{1}, u_{2} \in W_{1}\left(M, T_{*}\right) .
$$

Then $v=u_{1}-u_{2}$ satisfies

$$
\begin{aligned}
& \int_{0}^{1} v^{\prime \prime} w d x+\int_{0}^{1}\left(\lambda_{0} v_{x}+\lambda_{1} v_{x}^{\prime}+v_{x}^{\prime \prime}\right) w_{x} d x \\
& -\lambda \int_{0}^{1} \int_{0}^{\infty} g(s) v_{x}(t-s) d s w_{x} d x \\
& =\int_{0}^{1}\left(F\left[u_{1}\right]-F\left[u_{2}\right]\right) w d x+\int_{0}^{1}\left(F\left[u_{1}\right]-F\left[u_{2}\right]\right) w_{x} d x
\end{aligned}
$$

for all test function $w \in H_{0}^{1}$, for almost all $t \in[0, T]$. Taking $v^{\prime}=w$ in 2.39$)_{1}$ and integrating with respect to $t$, for

$$
\begin{aligned}
& e(t)=\int_{0}^{1}\left[\left|w^{\prime}\right|^{2}+\left(\lambda_{0}-\lambda \int_{0}^{\infty} g(s) d s\right)\left|w_{x}\right|^{2}+\left|w_{x}^{\prime}\right|^{2}\right] d x \\
& +\lambda \int_{0}^{\infty} g(s) \int_{0}^{1}\left|w_{x}(t-s)-w_{x}(t)\right|^{2} d x d s \\
& +2 \lambda_{1} \int_{0}^{t} \int_{0}^{1}\left|w_{x}^{\prime}\right|^{2} d x d s-\lambda \int_{0}^{t} \int_{0}^{\infty} g^{\prime}(s) \int_{0}^{1}\left|w_{x}(t-s)-w_{x}(t)\right|^{2} d x d s d \tau
\end{aligned}
$$


we obtain

$$
e(t)=\int_{0}^{1}\left(F\left[u_{1}\right]-F\left[u_{2}\right]\right) v^{\prime} d x+\int_{0}^{1}\left(F\left[u_{1}\right]-F\left[u_{2}\right]\right) v_{x}^{\prime} d x
$$

On the other hand, by (Hyp2)-(Hyp4), we deduce from (2.3), that

$$
\int_{0}^{1}\left|F[u-1]-F\left[u_{2}\right]\right| d x \leq 2 c(1+2 M) \bar{F}_{M} e^{1 / 2}(s) .
$$

Then

$$
E(t) \leq\left[4 c(1+2 M) \bar{F}_{M} \int_{0}^{t} e(s) d s .\right.
$$

Thanks again to Gronwall's Lemma, we have $E \equiv 0$, i.e., $u_{1} \equiv u_{2}$.

Theorem 2.3 is completely proved.

\section{BLOW UP}

We further prove that if 3.10 hold, then the blow up of any weak solution (3.1) for a finite time occurs when the initial energy is negative.

Here, we consider $1.1-1.4$ with $f=0, f_{1}=F\left(t, x, u, u_{x}\right) \in C^{1}\left(\mathbb{R}^{2} ; \mathbb{R}\right), f_{2}=$ $F\left(t, x, u, u_{x}\right) \in C^{1}\left(\mathbb{R}^{2} ; \mathbb{R}\right)$ as follows

$$
\left\{\begin{array}{l}
u^{\prime \prime}-\left(\lambda_{0} u_{x}+\lambda_{1} u_{x}^{\prime}+u_{x}^{\prime \prime}\right)_{x}+\lambda \int_{-\infty}^{t} g(t-s) u_{x x}(s) d s \\
=f_{1}\left(u, u_{x}\right)-\left(f_{2}\left(u, u_{x}\right)\right)_{x}, \quad x \in(0,1), 0<t<T_{*},
\end{array}\right.
$$

with the boundary conditions

$$
u(0, t)=u(1, t)=0, \quad t>0,
$$

and the following initial conditions:

$$
u(x,-t)=u_{0}(x, t), \quad u^{\prime}(x, 0)=u_{1}(x),
$$

We have proved in the previous section, the existence of local weak solution of (1.1)-1.4 in the Theorem 2.3 Furthermore, let us assume that there exist $\mathcal{F} \in$ $C^{2}\left(\mathbb{R}^{2} ; \mathbb{R}\right)$ and the constants $p, q>2 ; d_{1}, d_{2}>0$, such that

$$
\begin{aligned}
& \frac{\partial \mathcal{F}}{\partial u}(u, v)=f_{1}(u, v), \quad \frac{\partial \mathcal{F}}{\partial v}(u, v)=f_{2}(u, v), \\
& u f_{1}(u, v)+v f_{2}(u, v) \geq d_{1} \mathcal{F}(u, v), \quad \forall(u, v) \in \mathbb{R}^{2}, \\
& \mathcal{F}(u, v) \geq d_{2}\left(|u|^{p}+|v|^{q}\right), \quad \forall(u, v) \in \mathbb{R}^{2}
\end{aligned}
$$

We introduce the energy functional $E(t)$ associated with system $3.1-3.3$

$$
\begin{aligned}
& E(t)=\frac{1}{2} \int_{0}^{1}\left|u^{\prime}\right|^{2} d x+\frac{1}{2} \int_{0}^{1}\left(\lambda_{0}-\lambda \int_{0}^{\infty} g(s) d s\right)\left|u_{x}\right|^{2} d x+\frac{1}{2} \int_{0}^{1}\left|u_{x}^{\prime}\right|^{2} d x \\
& +\frac{1}{2} \lambda \int_{0}^{1} \int_{0}^{\infty} g(s)\left|u_{x}(t)-u_{x}(t-s)\right|^{2} d s d x-\int_{0}^{1} \mathcal{F}\left(u, u_{x}\right) d x
\end{aligned}
$$

It is note hard to see this Lemma (Using Lemma 2.2. 
Lemma 3.1. Suppose that (Hyp1) holds. Let u be solution of system (3.1)-(3.3). Then the energy functional (3.5) is a non-increasing function, i.e., for all $t \geq 0$,

$$
\frac{d}{d t} E(t)=-\lambda_{1} \int_{0}^{1}\left|u_{x}^{\prime}\right|^{2} d x+\frac{1}{2} \lambda \int_{0}^{1} \int_{0}^{\infty} g^{\prime}(s)\left|u_{x}(t)-u_{x}(t-s)\right|^{2} d s d x .
$$

For reader, we state this Lemma with its proof.

Lemma 3.2. Let $\nu>0$ be a real positive number and let $L(t)$ be a solution of the ordinary differential inequality

$$
\frac{d L(t)}{d t} \geq \xi L^{1+\nu}(t)
$$

defined in $[0, \infty)$. If $L(0)>0$, then the solution does not exist for $t \geq L(0)^{-\nu} \xi^{-\nu} \nu^{-1}$.

Proof. The direct integration of (3.7) gives

$$
L^{-\nu}(0)-L^{-\nu}(t) \geq \xi \nu t
$$

Thus, we get the following estimate:

$$
L^{\nu}(t) \geq\left[L^{-\nu}(0)-\xi \nu t\right]^{-1} .
$$

It is clear that the right-hand side of $(3.8)$ is unbounded for

$$
\xi \nu t=L^{-\nu}(0) .
$$

Lemma 3.2 is proved.

Our goal is to prove that when the initial energy is negative, the solution of system (3.1) blows up in finite time under the (3.4), (3.10). The regularity obtained by (2.2) implies that problem (1.1)-(1.4) admits a unique strong solution

$$
u \in C^{1}\left(\left[0, T_{*}\right] ; H_{0}^{1} \cap H^{2}\right), \quad u^{\prime \prime} \in L^{\infty}\left(0, T_{*} ; H_{0}^{1} \cap H^{2}\right) .
$$

Second main theorem. Our result here reads as follows.

Theorem 3.3. Assume that (3.4) hold. Assume further that $E(0)<0$ for any $u_{0}(0), u_{1} \in H_{0}^{1} \cap H^{2}$ hold. There exist a number $r, 2<r<\min \{p, q\}, r<d_{1} d_{2}$ such that

$$
\int_{0}^{\infty} g(s) d s<\frac{\lambda_{0}(1+r / 2)}{\lambda(1 / 2 r+1+r / 2)} .
$$

Then, the unique weak solution u of (3.1)-(3.3) blows up in finite time.

Proof. Let

$$
H(t)=-E(t), \quad \forall t \in\left[0, T_{*}\right) .
$$

By multiplying the equation in (3.1) by $-u^{\prime}$, integrating over $[0,1]$ and using Lemma 2.2 , we obtain

$$
\begin{aligned}
-\frac{d}{d t} H(t) & =\lambda_{1} \int_{0}^{1}\left|u_{x}^{\prime}\right|^{2} d x-\frac{1}{2} \lambda \int_{0}^{1} \int_{0}^{\infty} g^{\prime}(s)\left|u_{x}(t)-u_{x}(t-s)\right|^{2} d s d x \\
& \geq 0, \quad \forall t \in\left[0, T_{*}\right) .
\end{aligned}
$$


Consequently, $E(0)<0$, we have

$$
\begin{aligned}
& H(0)=-\frac{1}{2} \int_{0}^{1}\left|u_{1}\right|^{2} d x-\frac{1}{2} \int_{0}^{1}\left(\lambda_{0}-\lambda \int_{0}^{\infty} g(s) d s\right)\left|u_{0 x}(0)\right|^{2} d x-\frac{1}{2} \int_{0}^{1}\left|u_{1 x}\right|^{2} d x \\
& -\lambda \int_{0}^{1} \int_{0}^{\infty} g(s)\left|u_{0 x}(0)-u_{0 x}(-s)\right|^{2} d s d x+\int_{0}^{1} \mathcal{F}\left(u_{0}(x, 0), u_{0 x}(x, 0)\right) d x,
\end{aligned}
$$

inequality 3.12 implies

$$
0<H(0) \leq H(t), \quad \forall t \in\left[0, T_{*}\right) .
$$

Using (Hyp1), to get

$$
\begin{aligned}
H(t)-\int_{0}^{1} \mathcal{F}\left(u, u_{x}\right) d x & =-\frac{1}{2} \int_{0}^{1}\left|u^{\prime}\right|^{2} d x-\frac{1}{2} \int_{0}^{1}\left(\lambda_{0}-\lambda \int_{0}^{\infty} g(s) d s\right)\left|u_{x}\right|^{2} d x \\
& -\lambda \int_{0}^{1} \int_{0}^{\infty} g(s)\left|u_{x}(t)-u_{x}(t-s)\right|^{2} d s d x-\frac{1}{2} \int_{0}^{1}\left|u_{x}^{\prime}\right|^{2} d x \\
& \leq 0, \quad \forall t \in\left[0, T_{*}\right) .
\end{aligned}
$$

One implies

$$
0<H(0) \leq H(t) \leq \int_{0}^{1} \mathcal{F}\left(u, u_{x}\right) d x .
$$

Then, we define a functionals

$$
\begin{aligned}
M(t) & =\frac{1}{2} \int_{0}^{1} u^{2} d x \\
N(t) & =\lambda_{1} \frac{1}{2} \int_{0}^{1}\left|u_{x}\right|^{2} d x+\int_{0}^{1} u_{x} u_{x}^{\prime} d x
\end{aligned}
$$

and introduce

$$
L(t)=H^{1-\sigma}(t)+\varepsilon M^{\prime}(t)+\varepsilon N(t),
$$

for $\varepsilon$ small enough and

$$
0<\sigma<1,2 /(1-2 \sigma) \leq \min \{p, q\} .
$$

We now show that $L(t)$ satisfies the differential inequality in Lemma 3.2 By taking the derivative of (3.18) and using (3.1) and Lemma 2.2, we obtain

$$
\begin{aligned}
L^{\prime}(t) & =(1-\sigma) H^{-\sigma}(t) H^{\prime}(t)+\varepsilon \int_{0}^{1}\left|u^{\prime}\right|^{2} d x+\varepsilon \int_{0}^{1}\left|u_{x}^{\prime}\right|^{2} d x \\
& -\varepsilon\left(\lambda_{0}-\lambda \int_{0}^{\infty} g(s) d s\right) \int_{0}^{1}\left|u_{x}\right|^{2} d x \\
& +\varepsilon \lambda \int_{0}^{\infty} g(s) \int_{0}^{1}\left[u_{x}(t-s)-u_{x}(s)\right] u_{x} d x d s \\
& +\varepsilon \int_{0}^{1} f_{1}\left(u, u_{x}\right) u d x+\varepsilon \int_{0}^{1} f_{2}\left(u, u_{x}\right) u_{x} d x
\end{aligned}
$$

By the Cauchy-Schwarz and Young inequalities, we find

$$
\int_{0}^{\infty} g(s) \int_{0}^{1}\left[u_{x}(t-s)-u_{x}(s)\right] u_{x} d x d s
$$




$$
\begin{aligned}
& \leq \int_{0}^{\infty} g(s) \int_{0}^{1}\left|u_{x}(t-s)-u_{x}(s)\right|^{2} d x \int_{0}^{1}\left|u_{x}\right|^{2} d x d s \\
& \leq \gamma \int_{0}^{\infty} g(s) \int_{0}^{1}\left|u_{x}(t-s)-u_{x}(s)\right|^{2} d x d s+\frac{\int_{0}^{\infty} g(s) d s}{4 \gamma} \int_{0}^{1}\left|u_{x}\right|^{2} d x, \gamma>0 .
\end{aligned}
$$

Therefore,

$$
\begin{aligned}
L^{\prime}(t) & \geq(1-\sigma) H^{-\sigma}(t) H^{\prime}(t)+\varepsilon \int_{0}^{1}\left|u^{\prime}\right|^{2} d x+\varepsilon \int_{0}^{1}\left|u_{x}^{\prime}\right|^{2} d x \\
& -\varepsilon\left(\lambda_{0}-\lambda \int_{0}^{\infty} g(s) d s-\lambda \frac{\int_{0}^{\infty} g(s) d s}{4 \gamma}\right) \int_{0}^{1}\left|u_{x}\right|^{2} d x \\
& +\varepsilon \lambda \gamma \int_{0}^{\infty} g(s) \int_{0}^{1}\left|u_{x}(t-s)-u_{x}(s)\right|^{2} d x d s \\
& +\varepsilon \int_{0}^{1} f_{1}\left(u, u_{x}\right) u d x+\varepsilon \int_{0}^{1} f_{2}\left(u, u_{x}\right) u_{x} d x .
\end{aligned}
$$

By (3.4), we obtain

$$
\begin{gathered}
\int_{0}^{1} f_{1}\left(u, u_{x}\right) u d x+\int_{0}^{1} f_{2}\left(u, u_{x}\right) u_{x} d x \\
\geq d_{1} \int_{0}^{1} \mathcal{F}\left(u, u_{x}\right) d x \\
\int_{0}^{1} \mathcal{F}\left(u, u_{x}\right) d x \geq d_{2}\left(\int_{0}^{1}\left|u_{x}(t)\right|^{q} d x+\int_{0}^{1}|u(t)|^{p} d x\right) .
\end{gathered}
$$

Then, it follows since 3.19 and due to the inequality

$$
(1-\sigma) H^{-\sigma}(t) H^{\prime}(t)>0, \forall t \in\left[0, T_{*}\right),
$$

that

$$
\begin{aligned}
L^{\prime}(t) & \geq \varepsilon \int_{0}^{1}\left|u^{\prime}\right|^{2} d x+\varepsilon \int_{0}^{1}\left|u_{x}^{\prime}\right|^{2} d x \\
& -\varepsilon\left(\lambda_{0}-\lambda \int_{0}^{\infty} g(s) d s+\lambda \frac{\int_{0}^{\infty} g(s) d s}{4 \gamma}\right) \int_{0}^{1}\left|u_{x}\right|^{2} d x \\
& +\varepsilon \lambda \gamma \int_{0}^{\infty} g(s) \int_{0}^{1}\left|u_{x}(t-s)-u_{x}(s)\right|^{2} d x d s \\
& +\varepsilon d_{1} d_{2}\left(\int_{0}^{1}\left|u_{x}(t)\right|^{q} d x+\int_{0}^{1}|u(t)|^{p} d x\right) .
\end{aligned}
$$

Adding and substituting $r E(t), 2<r<\min \{p, q\}$; by using the definition of $H(t)$, to get

$$
\begin{aligned}
L^{\prime}(t) & \geq \varepsilon\left(1+\frac{r}{2}\right) \int_{0}^{1}\left|u^{\prime}\right|^{2} d x+\varepsilon\left(1+\frac{r}{2}\right) \int_{0}^{1}\left|u_{x}^{\prime}\right|^{2} d x+\varepsilon r H(t) \\
& -\varepsilon\left[\left(\lambda_{0}-\lambda \int_{0}^{\infty} g(s) d s+\lambda \frac{\int_{0}^{\infty} g(s) d s}{4 \gamma}\right)\right. \\
& \left.+\frac{r}{2}\left(\lambda_{0}-\lambda \int_{0}^{\infty} g(s) d s\right)\right] \int_{0}^{1}\left|u_{x}\right|^{2} d x
\end{aligned}
$$




$$
\begin{aligned}
& +\varepsilon \lambda\left(\frac{r}{2}-\gamma\right) \int_{0}^{\infty} g(s) \int_{0}^{1}\left|u_{x}(t-s)-u_{x}(s)\right|^{2} d x d s \\
& +\varepsilon\left(d_{1} d_{2}-r\right)\left(\int_{0}^{1}\left|u_{x}(t)\right|^{q} d x+\int_{0}^{1}|u(t)|^{p} d x\right)
\end{aligned}
$$

for some

$$
\begin{gathered}
a_{1}=\frac{r}{2}-\gamma>0, \quad \text { i.e. } \gamma<r / 2 \\
a_{2}=\lambda_{0}\left[-1+\frac{r}{2}\right]-\lambda\left[\frac{1}{4 \gamma}-1+\frac{r}{2}\right] \int_{0}^{\infty} g(s) d s>0 . \\
a_{3}=d_{1} d_{2}-r>0 .
\end{gathered}
$$

Then, estimate (3.24) becomes

$$
\begin{aligned}
L^{\prime}(t) & \geq \varepsilon\left(1+\frac{r}{2}\right) \int_{0}^{1}\left|u^{\prime}\right|^{2} d x+\varepsilon\left(1+\frac{r}{2}\right) \int_{0}^{1}\left|u_{x}^{\prime}\right|^{2} d x+\varepsilon p H(t) \\
& +\varepsilon \lambda a_{2} \int_{0}^{1}\left|u_{x}\right|^{2} d x+\varepsilon \lambda a_{1} \int_{0}^{\infty} g(s) \int_{0}^{1}\left|u_{x}(t)-u_{x}(t-s)\right|^{2} d x d s \\
& +\varepsilon a_{3}\left(\int_{0}^{1}\left|u_{x}(t)\right|^{q} d x+\int_{0}^{1}|u(t)|^{p} d x\right) .
\end{aligned}
$$

At this point,we can find positive constants $\nu>0$ such that

$$
\begin{array}{r}
L^{\prime}(t) \geq \varepsilon \nu\left[H(t)+\int_{0}^{1}\left|u_{x}(t)\right|^{q} d x+\int_{0}^{1}|u(t)|^{p} d x+\int_{0}^{1}\left|u_{x}\right|^{2} d x\right. \\
\left.+\int_{0}^{\infty} g(s) \int_{0}^{1}\left|u_{x}(t)-u_{x}(t-s)\right|^{2} d x d s+\int_{0}^{1}\left|u_{x}^{\prime}\right|^{2} d x+\int_{0}^{1}\left|u_{x}^{\prime}\right|^{2} d x\right] .
\end{array}
$$

Thus, we can choose $\varepsilon>0$ small enough such that

$$
L(t) \geq L(0)>0, \quad \forall t \in\left[0, T_{*}\right) .
$$

Further, by (3.19), Holder and Young inequalities, we obtain

$$
\left(\int_{0}^{1} u u^{\prime} d x\right)^{1 /(1-\sigma)} \leq c\left(\left(\int_{0}^{1}|u|^{p} d x\right)^{\tau / p(1-\sigma)}+\left(\int_{0}^{1}\left|u^{\prime}\right|^{2} d x\right)^{s / 2(1-\sigma)}\right)
$$

for $\frac{1}{\tau}+\frac{1}{s}=1$. We take $s=2(1-\sigma)$ to get

By using the algebraic inequality

$$
\frac{\tau}{1-\sigma}+\frac{2}{1-2 \sigma} \text {. }
$$

$$
z^{\nu} \leq(z+1) \leq\left(1+\frac{1}{a}\right)(z+a), \quad \forall z \geq 0, \quad 0<\nu \leq 1, a \geq 0,
$$

we find

$$
\left(\int_{0}^{1}|u|^{p} d x\right)^{2 / p(1-2 \sigma)} \leq c\left(\int_{0}^{1}|u|^{p} d x+H(t)\right), \quad \forall t \in\left[0, T_{*}\right) .
$$

Then

$$
\left(\int_{0}^{1} u u^{\prime} d x\right)^{1 /(1-\sigma)} \leq c\left(\int_{0}^{1}|u|^{p} d x+\int_{0}^{1}\left|u^{\prime}\right|^{2} d x+H(t)\right) .
$$

Similarly, we obtain

$$
\left(\int_{0}^{1} u_{x} u_{x}^{\prime} d x\right)^{1 /(1-\sigma)} \leq c\left(\int_{0}^{1}\left|u_{x}\right|^{q} d x+\int_{0}^{1}\left|u_{x}^{\prime}\right|^{2} d x+H(t)\right) .
$$


Moreover, we note that

$$
\begin{aligned}
L^{1 /(1-\sigma)}(t) & \leq c\left[\left(H^{1-\sigma}(t)+\int_{0}^{1} u u^{\prime} d x\right)^{1 /(1-\sigma)}+\left.\left.\left|\int_{0}^{1}\right| u_{x}\right|^{2} d x\right|^{1 /(1-\sigma)}+\left|\int_{0}^{1} u_{x} u_{x}^{\prime} d x\right|^{1 /(1-\sigma)}\right], \\
& \leq\left[H(t)+\int_{0}^{1}|u|^{p} d x+\int_{0}^{1}\left|u^{\prime}\right|^{2} d x+\int_{0}^{1}\left|u_{x}\right|^{2} d x+\int_{0}^{1}\left|u_{x}\right|^{q} d x+\int_{0}^{1}\left|u_{x}^{\prime}\right|^{2} d x\right] .
\end{aligned}
$$

This yields

$$
L^{\prime}(t) \geq a_{0} L^{1 /(1-\sigma)(t)}, \quad \forall t \geq 0 .
$$

Finally, as a result of simple integration of $(3.30)$, completes the proof of Theorem 3.3 .

\section{REFERENCES}

[1] J. Ball; Remarks on blow up and nonexistence theorems for nonlinear evolutions equations, Quart. J. Math. Oxford, 28 (1977), 473-486.

[2] A. Beniani, A. Benaissa and Kh. Zennir Polynomial Decay of Solutions to the Cauchy Problem for a Petrovsky-Petrovsky System in $\mathbb{R}^{n}$, Acta. Appl. Math., 146, 1 (2016), 67-79.

[3] A. Braik, A. Beniani and Kh. Zennir Polynomial stability for system of 3 wave equations with infinite memories, Math. Meth. Appl. Sci. 2017; 115. DOI: 10.1002/mma.4599

[4] A. Braik, Y. Miloudi and Kh. Zennir A finite-time blow-up result for a class of solutions with positive initial energy for coupled system of heat equations with memories, Math. Meth. Appl. Sci. 2017; 19. DOI: 10.1002/mma.4695

[5] E. L. A. Coddington, N. Levinson; Theory of ordinary differential equations, McGraw-Hill, 1955, p. 43.

[6] M. M. Cavalcanti, U V. N. Domingos Cavalcanti, J. S. Prates Filho; Existence and exponential decay for a Kirchhoff Carrier model with viscosity, J. Math. Anal. Appl. 226 (1998), 40-60.

[7] M. M. Cavalcanti, U V. N. Domingos Cavalcanti, I. Lasieka; Well-posedness and optimal decay rates for the wave equation with nonlinear boundary damping-source interaction, J. Differ. Eq. (236), (2007), 407-459.

[8] M. M. Cavalcanti, U V. N. Domingos Cavalcanti, J. A. Soriano; Global solvability and asymptotic stability for the wave equation with nonlinear boundary damping and source term, Progress in Nonli. Diff. Equ. Appl. 66 (2005), 161-184.

[9] C. M. Dafermos, On the existence and the asymptotic stability of solution to the equations of linear thermo-elasticity, Arch. Ration. Mech. Anal., 29, (1968) 241-271.

[10] C. M. Dafermos, Asymptotic stability in visco-elasticity, Arch. Ration. Mech. Anal. 37(1970), 297-308.

[11] C. Giorgi, J. Muenoz Rivera and V. Pata, Global Attractors for a Semilinear Hyperbolic Equation in visco-elasticity, J. Math. Anal. Appl., 260(1), (2001) 83-99.

[12] X. Han and M. Wang, Global existence and blow-up of solutions for a system of nonlinear visco-elastic wave equations with damping and source, Nonli. Anal., 71(2009), 5427-5490.

[13] W. Hrusa, Global existence and asymptotic stability for a semi-linear Volterra equation with large initial data, SIAMJ. Math. Anal. 16(1)(1985), 110-134.

[14] I. Lasiecka and D. Tataru, Uniform boundary stabilization of semi-linear wave equations with nonlinear boundary damping, Diff. Integ. Equ., 6 (3), 1993, 507-533.

[15] Mohammad M. Al-Gharabli, A general decay result of a visco-elastic equation with infinite history and nonlinear damping, Applicable Analysis, 97:3, 382-399, DOI: 10.1080/00036811.2016.1268686.

[16] M. Mustafa, Well posedness and asymptotic behavior of a coupled system of nolinear viscoelastic equations, Nonlin. Anal. (13), (2012), 452-463.

[17] Jianghao Hao, Peipei Wang; General Stability Result of Abstract Thermoelastic System with Infinite Memory, Bull. Malays. Math. Sci. Soc. (2018). https://doi.org/10.1007/s40840-0180615-z.

[18] S. A. Messaoudi; Blow up and global existence in nonlinear visco-elastic wave equations, Math. Nachrich. 260 (2003), 58-66.

[19] S. A. Messaoudi; Blow up of solutions with positive initial energy in a nonlinear visco-elastic wave equations, J. Math. Anal. Appl. 320 (2006), 902-915. 
[20] S. A. Messaoudi; General decay of solutions of a visco-elastic equation, J. Math. Anal. Appl. 341(2) (2008), 1457-1467.

[21] S. A. Messaoudi, Muhammad M. Al-Gharabli; A general stability result for a nonlinear wave equation with infinite memory, Applied Mathematics Letters, 26 (2013) 1082-1086

[22] L. T. P. Ngoc, N. T. Duy, N. T. Long; A linear recursive scheme associated with the Love's equation, Acta Mathematica Vietnamica, 38 (4) (2013), 551-562.

[23] L. T. P. Ngoc, N. T. Duy, N. T. Long; On a high-order iterative scheme for a nonlinear Love equation, Applications of Mathematics, 60 (3)(2015), 285-298.

[24] L. T. P. Ngoc, N. T. Long; Existence, blow-up and exponential decay for a nonlinear Love equation associated with Dirichlet conditions, Applications of Mathematics, 61(2) (2016), 165-196.

[25] Kosuke Ono; On global solutions and blow-up solutions of nonlinear Kirchhoff strings with nonlinear dissipation, J.Math. Anal. Appl. 216 (1997), 321-342.

[26] Fenglong Sun, Lishan Liu and Yonghong Wu; Blow-up of solutions for a nonlinear viscoelastic wave equation with initial data at arbitrary energy level, Applicable Analysis, DOI: 10.1080/00036811.2018.1460812

[27] Sun-Hye PARK; Stability of a von karman equation with infinite memory, Acta Mathematica Scientia 2017,37B(4):965-973

[28] L. X. Truong, L. T. P. Ngoc, A. P. N. Dinh, N. T. Long, Existence, blow-up and exponential decay estimates for a nonlinear wave equation with boundary conditions of two-point type, Nonlinear Anal. TMA. 74 (18) (2011), 6933-6949.

[29] N. A. Triet, V. T. T. Mai, L. T. P. Ngoc, N. T. Long, A Dirichlet problem for a nonlinear wave equation of Kirchhoff - Love type, Nonlinear Functional Analysis and Applications, 22 (3) (2017), 595-626.

[30] N. A. Triet, V. T. T. Mai, L. T. P. Ngoc, N. T. Long, Existence, blow-up and exponential decay for Kirchhoff-love equations with Dirichlet conditions, Electronic Journal of Differential Equations, Vol. 2018 (2018), No. 167, pp. 1-26.

[31] Wenjun Liu and H. Zhuang; Global existence, asymptotic behavior and blow-up of solutions for a suspension bridge equation with nonlinear damping and source terms, Nonlinear Differ. Equ. Appl., (2017) 24: 67. https://doi.org/10.1007/s00030-017-0491-5

[32] Z. Yang, Z. Gong; Blow-up of solutions for visco-elastic equations of Kirchhoff type with arbitrary positive initial energy, Elect. J. Diffe. Equ., Vol. 2016 (2016), No. 332, pp. 1-8.

[33] Kh. zennir, Global nonexistence of Solutions for a system of nonlinear visco-elastic wave equations with degenerate damping and source terms. Ukra. Math. J. 67, No 7, (2013) 723739.

[34] Kh. zennir, General decay of solutions for damped wave equation of Kirchhoff type with density in $\mathbb{R}^{n}$. Ann. Univ. Ferrara 61(2015) 381-394.

[35] S. Zitouni, and Kh. Zennir, On the existence and decay of solution for visco-elastic wave equation with nonlinear source in weighted spaces. Rend. Circ. Mat. Palermo, II. Ser, Vol. 66, No 3 (2017), pp 337-353.

KHALED ZENNiR

First address: Department of mathematics, University 20 Août 1955- Skikda, 21000, Algeria

Second address: Department of Mathematics, College of Sciences and Arts, Al-Ras, QAssim University, Kingdom of Saudi Arabia.

E-mail address: khaledzennir4@yahoo.vom 